\title{
Nefropatía no amiloidea por depósito de inmunoglobulinas monoclonales A propósito de un caso de enfermedad idiopática por depósito de cadenas ligeras
}

\author{
Jorge de Jesús Cantillo ${ }^{1,2}$, Rocío del Pilar López ${ }^{3,4}$, Rafael Enrique Andrade ${ }^{3,4,5}$ \\ 1 Servicio de Nefrología y Diálisis, Hospital El Tunal, E.S.E., Bogotá, D.C., Colombia. \\ 2 Servicio de Terapia Renal, Hospital Universitario Clínica San Rafael, Bogotá, D.C., Colombia. \\ 3 Departamento de Patología y Laboratorios, Hospital Universitario Fundación Santa Fe de Bogotá, Bogotá, \\ D.C., Colombia. \\ ${ }^{4}$ Facultad de Medicina, Universidad de los Andes, Bogotá, D.C., Colombia. \\ ${ }_{5}$ Facultad de Medicina, Universidad Nacional de Colombia, Bogotá, D.C., Colombia.
}

La nefropatía de las gammapatías monoclonales es debida, principalmente, al depósito de cadenas ligeras. Las enfermedades renales paraproteinémicas son lesiones asociadas con depósitos de inmunoglobulinas intactas o fragmentos de inmunoglobulinas (cadenas pesadas y cadenas ligeras).

La enfermedad por depósito de cadenas ligeras es una condición rara, caracterizada por el depósito de cadenas monoclonales ligeras en muchos órganos y, en el riñón, predominantemente, en glomérulos y membranas basales tubulares.

La enfermedad está frecuentemente asociada con alteraciones linfoproliferativas y la mayoría de casos son causados por depósito de cadenas ligeras kappa. Aunque se presenta sobre todo en cuadros malignos, en ocasiones, no se detecta enfermedad hematológica y se denomina idiopática o primaria. Suele manifestarse como una insuficiencia renal grave con proteinuria nefrótica, no tiene tratamiento claramente establecido y el pronóstico es malo.

Se describen las características clínicas e histológicas del primer caso informado en Colombia de nefropatía por depósito de cadenas ligeras, diagnosticado en el contexto de una enfermedad renal paraproteinémica sin datos de malignidad.

Palabras clave: nefropatías, paraproteinemias, cadenas ligeras de inmunoglobulina, informes de casos.

Nonamyloidotic glomerular disease caused by light chain deposits: a case report

The nephropathy of monoclonal gammopathies is principally caused by light chain deposits of fragmented immunoglobins. Paraprotein-related renal diseases are associated with such deposits of intact (heavy chain) or fragmentary (light chain) immunoglobins. A condition of pathological light chain deposits is rare and characterized by deposits of fragments of monoclonal immunoglobulins in many organs. Renal deposits occur primarily in glomeruli and tubular basement membranes. This disease is frequently associated with lymphoproliferative disorders. The majority of cases are caused by deposits of kappa light chains. Whereas this disease is most frequently associated with hematologic malignancies, occasionally a case occurs without detectable hematological pathologies; these cases are called idiopathic or primary. They usually manifest themselves as severe renal insufficiencies with nephrotic-range proteinuria. No treatment regime has been clearly established and the prognosis is poor. Herein, the clinical and histological characteristics are described regarding the first case in Colombia of light chain deposit disease without symptoms of malignancy.

Key words: Kidney diseases, paraproteinemias, immunoglobulin light chains, case reports. 
Desde la primera descripción de amiloidosis por inmunoglobulinas por Glenner et al. en 1971 (1), el espectro de las enfermedades glomerulares por depósitos o precipitación de componentes de inmunoglobulina monoclonal ha crecido de forma notoria (2). Estas enfermedades pueden clasificarse en dos categorías con base en la microscopía electrónica (3).

La primera categoría es la de depósitos organizados, que incluye enfermedades de aspecto fibrilar, principalmente amiloidosis, y enfermedades con formaciones microtubulares, e incluye el riñón crioglobulinémico y la glomerulopatía "inmunotactoide".

La segunda categoría de enfermedades está caracterizada por depósitos granulares no organizados electrón-densos, que están localizados a lo largo de las membranas basales en la mayoría de los tejidos, especialmente, en los glomérulos y túbulos renales. La presencia de cadenas ligeras monoclonales en estas lesiones fue primero reconocida en 1973 por Antonovych et al. (4) y confirmada por Randall et al. (5), quienes publicaron en 1976, la primera descripción de enfermedad por depósito de cadenas ligeras.

Las gammapatías monoclonales son un conjunto de enfermedades que se caracterizan por la producción anómala y secreción a la sangre de una inmunoglobulina monoclonal (de un mismo clon de células plasmáticas) o un fragmento de la misma (cadenas pesadas, ligeras o ambas), que puede depositarse en los

\footnotetext{
* "We coined the diagnostic term immunotactoid glomerulopathy (ITG) to emphasize the morphology and the composition of the glomerular deposits that appeared as parallel microtubules by electron microscopy and that contained IgG and complement (C3) demonstrated by fluorescence microscopy."

Schwartz MM, Korbet SM, Lewis EJ. Immunotactoid Glomerulopathy. J Am Soc Nephrol. 2002:13:1390-7.
}

Correspondencia:

Jorge de Jesús Cantillo, Calle 114 A № 56-34, apartamento 601, Bogotá, D.C., Colombia

Teléfonos: (571) 714 4372, 560 2009, 5603284 y 5602464. md_cantillo@yahoo.com, md_cantillo@hotmail.com

Recibido: 03/04/09; aceptado:02/07/09 órganos de forma organizada, como cristales, fibrillas o microtúbulos, o de forma no organizada (granular). Esta inmunoglobulina se deposita, principalmente, en el riñón, no sólo porque es un órgano muy irrigado, sino también, porque el túbulo renal tiene un papel predominante en el metabolismo de las inmunoglobulinas (6-8).

El diagnóstico del compromiso renal debido al depósito de inmunoglobulinas se está ampliando con el desarrollo e implementación rutinaria de distintas técnicas de laboratorio: tinciones con anticuerpos específicos contra cadenas ligeras kappa y lambda, estudio con microscopía electrónica, desarrollo de técnicas cada vez más sensibles para detectar el componente monoclonal en sangre $u$ orina $(7,9)$.

La participación renal frecuentemente se asocia a gammapatías monoclonales malignas, generalmente asociadas a depósitos de cadenas ligeras. Tradicionalmente, se ha visto asociada a enfermedades como el mieloma - la macroglobulinemia de Waldesntrön (8). Sin embargo, el depósito renal de cadenas ligeras también se ha descrito en gammapatías monoclonales benignas (8). Existen entidades sin criterios de malignidad en las que es posible que las paraproteínas circulantes, ya sean cadenas ligeras, pesadas o ambas, muestren afinidad por el riñón, se depositen y sean causa de manifestaciones renales de síndrome nefrótico, proteinuria aislada 0 insuficiencia renal rápida y progresiva $(8,9)$. Por otro lado, es infrecuente que a lo largo del seguimiento estas presentaciones se tornen malignas (10).

La enfermedad por depósito de cadenas ligeras está caracterizada por el depósito generalizado de un solo tipo de cadena ligera a lo largo de la membrana basal del riñón. Generalmente, se describe en el curso de una discrasia de células plasmáticas u otra alteración linfoproliferativa pero, también, puede darse en ausencia de trastornos hematológicos y, en ese caso, se denomina enfermedad idiopática por depósito de cadenas ligeras (7)

Se describen las características clínicas e histológicas de un caso de nefropatía por depósito de cadenas ligeras en el contexto de 
una enfermedad renal paraproteinémica sin datos de malignidad.

\section{Presentación del caso}

Se trata de un hombre de 45 años de edad quien refirió malestar general desde dos meses antes de su hospitalización, hiporexia, astenia, adinamia, cefalea global pulsátil matinal de intensidad moderada, disminución de la agudeza visual y pérdida de peso $(3 \mathrm{~kg})$.

En la revisión por sistemas refirió náuseas, vómito ocasional, polidipsia, poliuria y orinas espumosas. Como antecedentes, interesaron el síndrome de Guillain-Barré (dos episodios: años 2000 y 2006), hipertensión arterial sistémica diagnosticada dos meses antes, ex fumador hasta hace dos años de 5 cigarrillos al día por 15 años; trabajó con madera y el padre sufre enfermedad renal crónica terminal que requiere diálisis crónica.

En el examen físico se encontró en regulares condiciones generales, con temperatura de $37^{\circ} \mathrm{C}$, tensión arterial de 159/100 mm Hg, frecuencia respiratoria de 20 por minuto, y frecuencia cardiaca de 100 por minuto. No hubo otros hallazgos positivos. Los exámenes paraclínicos se resumen en el cuadro 1. La ecografía renal y el ecocardiograma bidimensional con Doppler a color fueron normales, y la radiografía de tórax mostró signos de distensión pulmonar hipertensión pulmonar leve.

Como diagnósticos presuntivos se consideraron el síndrome nefrótico o nefrítico, la hipertensión arterial sistémica no controlada y la falla renal aguda.

Se iniciaron reajuste de la terapia antihipertensiva, profilaxis tromboembólica, antieméticos, gastroprotección y dieta hiposódica. Un día después de su admisión, se observó mejoría clínica paulatina y se ordenan exámenes para enfermedad glomerular (cuadro 1).

Diez días después de su ingreso, y considerando los hallazgos descritos, se practicó biopsia renal percutánea, en cuyo examen por microscopía de luz se observó: número de glomérulos, 39 (ninguno con esclerosis global y 5 con
Cuadro 1. Resultados de laboratorio clínico de ingreso.

\begin{tabular}{|c|c|}
\hline Glucemia & $130 \mathrm{mg} / \mathrm{dl}(70-109)$ \\
\hline Calcio sérico & $10 \mathrm{mg} / \mathrm{dl}(8,4-10,2)$ \\
\hline Cloro sérico & $112 \mathrm{mmol} / \mathrm{L}(98-107)$ \\
\hline Creatinina sérica & $1,9 \mathrm{mg} / \mathrm{dl}(0,7-1,3)$ \\
\hline BUN & $25 \mathrm{mg} / \mathrm{dL}(7-20)$ \\
\hline Potasio sérico & $5,1 \mathrm{mmol} / \mathrm{L}(3,5-5,1)$ \\
\hline Sodio sérico & $149 \mathrm{mmoL} / \mathrm{L}(136-145)$ \\
\hline Ácido úrico & $9,7 \mathrm{mg} / \mathrm{dl}(2,4-7)$ \\
\hline Fósforo sérico & $4,2 \mathrm{mg} / \mathrm{dl}(2,5-4,5)$ \\
\hline Magnesio sérico & $2,9 \mathrm{mg} / \mathrm{dl}(1,7-2,3)$ \\
\hline Albúmina sérica & $4,7 \mathrm{~g} / \mathrm{dl}$ \\
\hline Proteínas totales séricas & $6,4 \mathrm{~g} / \mathrm{dl}$ \\
\hline \multirow[t]{4}{*}{ Cuadro hemático } & $\begin{array}{l}\text { Hemoglogina: } 14 \mathrm{~g} / \mathrm{dl} \\
(12,2-18,1)\end{array}$ \\
\hline & $\begin{array}{l}\text { Hematócrito: } 39,7 \% \\
(37,7-53,7)\end{array}$ \\
\hline & $\begin{array}{l}\text { Plaquetas: } 256.000 x \\
\text { mm3 (142.000-424.000) }\end{array}$ \\
\hline & Leucocitos: 12.000 x mm3 \\
\hline PT & $10,5 \mathrm{seg} / 10 \mathrm{seg}(10,4-13)$ \\
\hline PTT & $25,9 \mathrm{seg} / 27,4 \mathrm{seg}(26-32)$ \\
\hline INR & 1,05 \\
\hline VSG & $38 \mathrm{~mm}$ por hora \\
\hline PCR cuantitativa & $2,3 \mathrm{mg} / \mathrm{dl}$ \\
\hline PCR ultra & $3,0 \mathrm{mg} / \mathrm{dl}$ \\
\hline Uroanálisis & $\begin{array}{l}\text { Proteínas: } 2.000 \mathrm{mg} / \mathrm{dl} \\
\text { Hematíes: } 65 \text { por campo }\end{array}$ \\
\hline \multicolumn{2}{|l|}{ Proteinuria en orina de } \\
\hline 24 horas & $0,38 \mathrm{~g}$ \\
\hline B2 microglobulina & 10.619 ng/ml (604-2.286) \\
\hline TSH & $0,876 \mathrm{ulU} / \mathrm{ml}(0,350-4,940)$ \\
\hline $\lg A$ & $68 \mathrm{mg} / \mathrm{dl}(63-484)$ \\
\hline $\lg G$ & 598 mg/dl (540-1.822) \\
\hline $\lg \mathrm{M}$ & $34 \mathrm{mg} / \mathrm{dl}(22-240)$ \\
\hline p-ANCA & Negativo \\
\hline C-ANCA & Negativo \\
\hline ANA & Negativos \\
\hline Anti ADN & Negativo \\
\hline C3 & 156 mg/dl (82-193) \\
\hline $\mathrm{C} 4$ & $42 \mathrm{mg} / \mathrm{dl}(15-57)$ \\
\hline HBsAg & Negativo \\
\hline Anti-HVC & Negativo \\
\hline Anti-VIH & Negativo \\
\hline
\end{tabular}

esclerosis segmentaria); proliferación celular endocapilar/del mesangio (+); engrosamiento difuso de las membranas basales capilares $(++)$; ensanchamiento del mesangio $(++)$; fibrosis túbulo-intersticial $(+)$; nefritis intersticial $(+)$; esclerosis arterial (de la subíntima) (+); media $(+)$; esclerosis arteriolar $(++)$ (media, ++ ); depóstio hialino en arteriolas aferentes y eferentes (+); estudio con rojo Congo, negativo para amiloide. 
Con inmunofluorescencia se encontró: IgG (++) lineal membrana basal capilar; IgM (++) pared vascular; C3 $(+)$ algunas paredes vasculares; cadenas ligeras kappa (+++) membrana basal capilar, membrana basal tubular y pared vascular lineal sin evidencia de reactividad para las cadenas ligeras lambda.

En la microscopía electrónica se encontró: engrosamiento difuso de la membrana basal capilar con presencia de dobles contornos e interposición del mesangio; depósitos electróndensos con apariencia de complejos inmunes

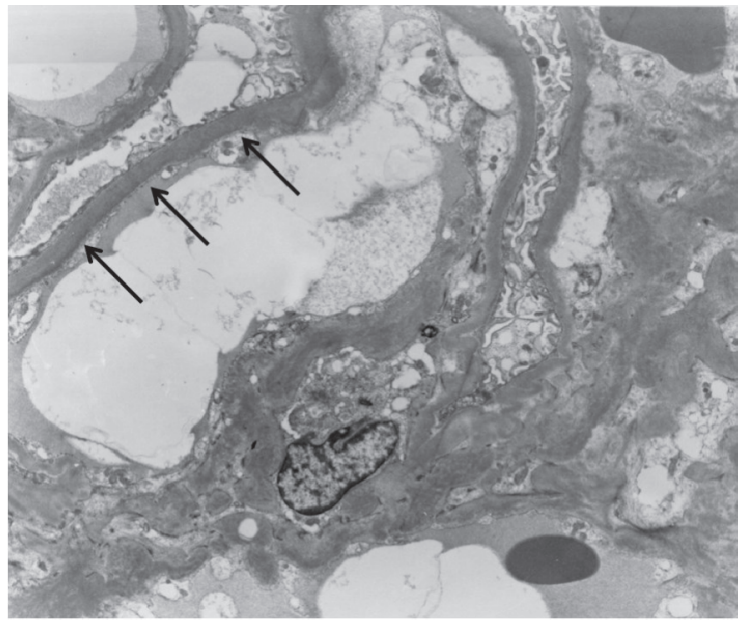

Figura 1. Presencia de depósitos electrón-densos a lo largo de la membrana basal capilar en localización subendotelial. Aumento original: 5.000X por microscopía electrónica.

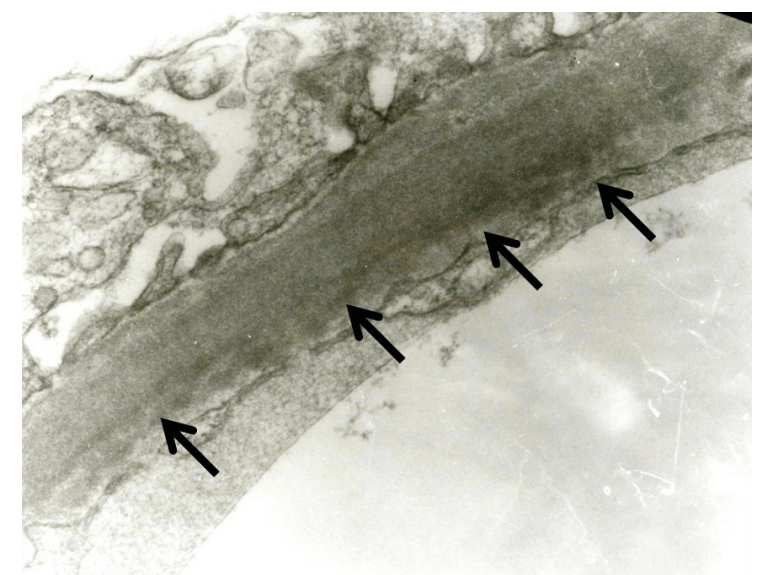

Figura 2. Presencia de depósitos electrón-densos a lo largo de la membrana basal capilar en localización subendotelial. Aumento original: 30.000X por microscopía electrónica. en localización subendotelial (+++), dispuestos masivamente en banda a lo largo de las mismas; en el mesangio había proliferación celular $(++)$ y expansión de la matriz (++) con presencia de material electrón-denso; frecuentes monocitos en la luz con fagolisosomas, y ausencia de otros depósitos, como amiloide, crioglobulinas, etc

Por anatomopatología se diagnosticó nefropatía de patrón membranoproliferativo, tipo enfermedad por depóstio de cadenas ligeras kappa (figuras 1-6).

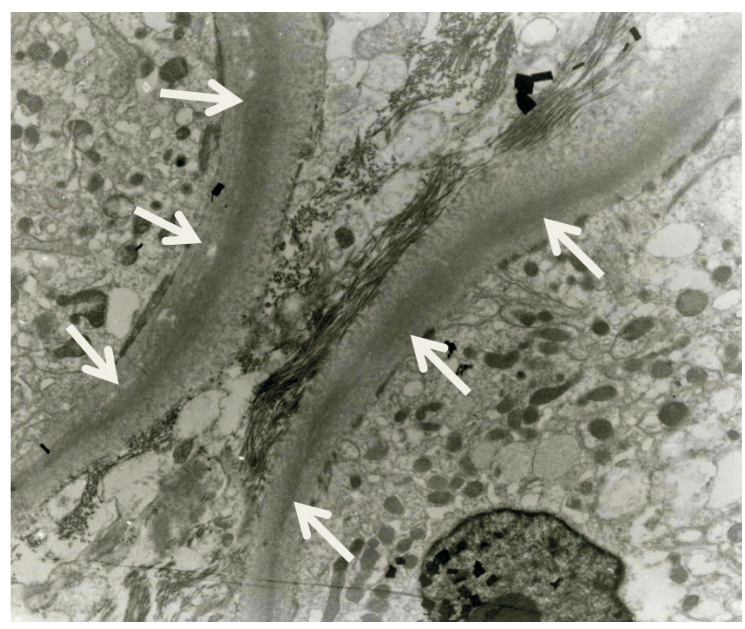

Figura 3. Presencia de depósitos electrón-densos a lo largo de la membrana basal capilar en localización intramembranosa. Aumento original: 4.000X por microscopía electrónica.

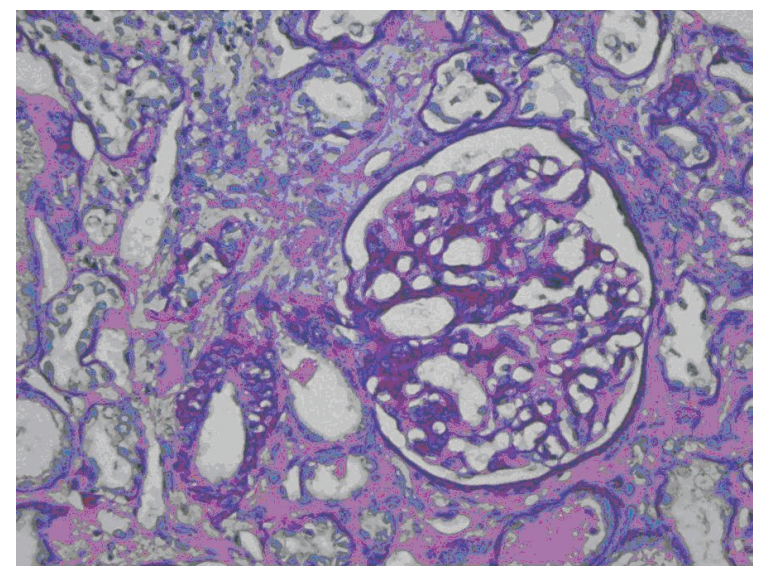

Figura 4. Glomérulo con engrosamiento difuso de membranas basales capilares y retracción de asas. Plata metenamina, 20X. 


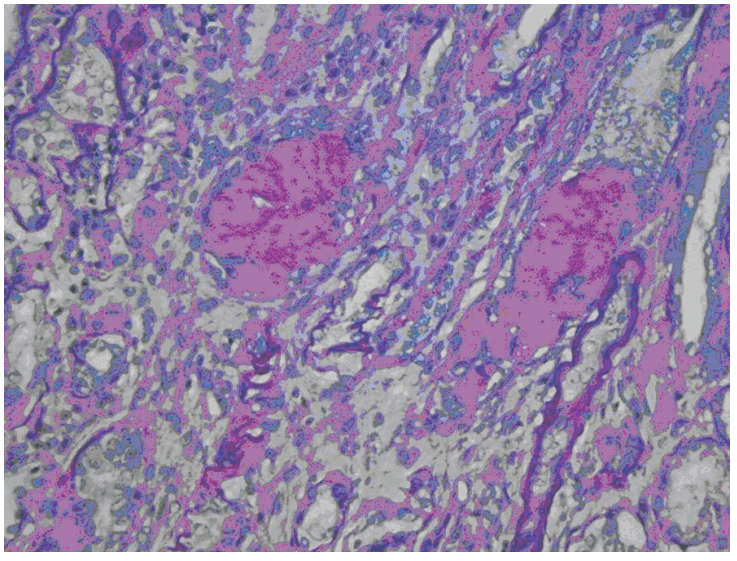

Figura 5. Cilindros densos intraluminales y engrosamiento difuso de membranas basales tubulares. PAS, 40X.

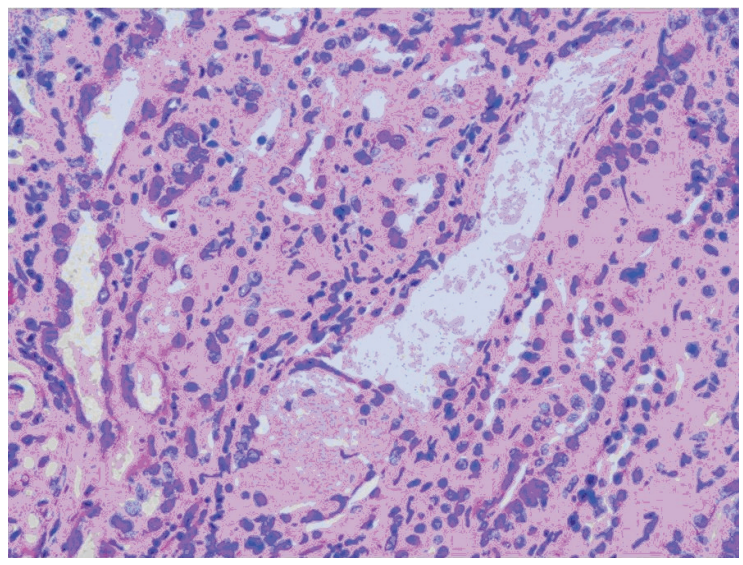

Figura 6. Cilindros densos intraluminales con reacción inflamatoria y rodeados por células fagocíticas. Inflamación intersticial crónica. Hematoxilina-eosina, 20X.

Casi un mes después de la práctica de la biopsia renal percutánea, el paciente fue admitido en el servicio de urgencias por emergencia hipertensiva, empeoramiento de la pérdida de peso, anemia (hemoglobina de $11 \mathrm{~g} / \mathrm{dl}$ ), astenia, adinamia, disminución de la agudeza visual y mayor deterioro de la función renal (creatinina de $3,8 \mathrm{mg} / \mathrm{dl}$, BUN de $53 \mathrm{mg} / \mathrm{dl}$ ).

Dado el resultado de la biopsia renal, se inició la evaluación hematológica. La biopsia de médula ósea mostró $70 \%$ de celularidad, relación entre mieloides y eritroides de 3:1, y ausencia de plasmocitos. Hubo representación de las líneas hematopoyéticas, de apariencia, número y ubicación normales. No se observó fibrosis ni infiltración neoplásica. La electroforesis de proteínas fue normal en suero y, en orina, no mostró bandas monoclonales. Los niveles séricos de cadenas livianas fueron de $1,64 \mathrm{mg} /$ dl $(1,38-3,75)$ para las kappa y de $0,52 \mathrm{mg} / \mathrm{dl}$ $(0,93-2,42)$ para las lambda. El estudio óseo no mostró lesiones líticas. Las inmunoglobulinas séricas fueron normales. Se encontró elevación de la microglobulina beta 2 .

Se practicaron también los siguientes estudios: biopsia gástrica, de colon y recto, sin evidencia de malignidad ni de depósito de amiloide; biopsia por aspiración de grasa abdominal subcutánea, negativa con tinción de rojo Congo; neuroconducción y electromiografía de las cuatro extremidades, temblor crónico por neuropatía. El paciente evolucionó hacia una insuficiencia renal rápida y progresiva, con síntomas urémicos y signos de hipervolemia (dos meses después de su primera hospitalización) e ingresó a un programa de terapia sustitutiva renal (diálisis).

\section{Discusión}

El presente caso clínico de enfermedad idiopática por depósito de cadenas ligeras en el contexto de una enfermedad renal paraproteinémica (lesiones renales asociadas con depósito de inmunoglobulina o fragmentos de inmunoglobulina) (11) es el primero que se informa en Colombia.

Las gammapatías monoclonales, aunque originadas por una proliferación anómala de células plasmáticas, no siempre responden a un proceso maligno, ni en el momento del diagnóstico ni en su posterior evolución (8). En la enfermedad por depósito de cadenas ligeras, los fragmentos de inmunoglobulina, típicamente tipo kappa, se acumulan con una conformación desorganizada granular, que no corresponde a la forma de depósito cristalino que se observa en el riñón con mieloma, a la fibrilar en la amiloidosis, ni a la microtubular en la glomerulopatía "inmunotactoide" (12).

La nefropatía por depósito de cadenas ligeras en el contexto de las gammapatías sin criterios hematológicos de malignidad, se ha descrito en 
casos anecdóticos, series cortas o como parte de cohortes más extensas junto con los casos de malignidad. En ocasiones, se ha mencionado su carácter sistémico o su asociación a neuropatía o hepatopatía (8). En $5 \%$ de las necropsias de pacientes con mieloma se ha encontrado compromiso renal por enfermedad por depósito de cadenas ligeras; sin embargo, la frecuencia con que se diagnostica la enfermedad es mucho más baja $(2,7)$.

En general, los datos de grandes series publicadas de enfermedad por depósito de inmunoglobulina monoclonal, muestran un rango amplio de edad en el momento de la presentación (26 a 94 años), con predilección por el sexo masculino $(2,3)$. En dos series de casos de enfermedad por depósito de cadenas ligeras publicadas en España, el análisis reveló una mayor frecuencia en mujeres y aparece en la edad media de la vida, aunque no puede descartarse en gente joven $(7,8)$.

Clínicamente, nos enfrentamos a un paciente en la edad media de la vida, con antecedentes neurológicos, hipertensión arterial sistémica de diagnóstico reciente, signos de enfermedad glomerular con presentación nefrótica-nefrítica (uroanálisis), síndrome constitucional y deterioro de la función renal (insuficiencia renal aguda o de rápida evolución) (7), con aumento al doble de la creatinina basal $(1,9 \mathrm{mg} / \mathrm{dl} \mathrm{a} 4,1 \mathrm{mg} / \mathrm{dl})$ en un corto período (mes y medio).

No asumimos una conducta terapéutica agresiva, porque una semana después de la admisión hospitalaria, la creatinemia se había reducido a 1,6 mg/dl, en comparación con 1,9 mg/dl al ingreso. Sin embargo, con los datos anteriores se sospechó inicialmente enfermedad glomerular en el contexto de una enfermedad sistémica: antecedentes neurológicos, hipertensión arterial sistémica de diagnóstico reciente, síndrome constitucional, signos de glomerulonefritis, gammapatías monoclonales e insuficiencia renal aguda.

El compromiso renal en la enfermedad por depósito de cadenas ligeras es constante, con insuficiencia renal, proteinuria e hipertensión arterial sistémica en la mayoría de los casos $(8,12)$. El síndrome nefrótico se presenta hasta en $55 \%$ de casos y, en el grupo de pacientes con proteinuria menor de $1 \mathrm{~g}$ al día, la clínica predominante es la de un síndrome túbulointersticial. Puede detectarse hematuria hasta en $40 \%$. La evolución hacia insuficiencia renal terminal suele ser rápida y con frecuencia comparable entre pacientes con diferentes grados de proteinuria $(2,12)$.

En la serie de casos clínicos de esta enfermedad publicada por Ramos et al., se encontró insuficiencia renal en $88 \%$ de los pacientes, de acuerdo con otras series en las que la insuficiencia renal oscilaba entre $92 \%$ y $96 \%$ en casos de enfermedad pura por depósito de inmunoglobulina monoclonal. Sin embargo, cerca de $90 \%$ de los pacientes presentaron inicialmente proteinuria, mientras que en otras series de la literatura fue sólo el $48 \%$ al $57 \%$ (8).

La presencia de proliferación endocapilar y del mesangio con la consiguiente expansión y engrosamiento difusos del mesangio, con aspecto de doble contorno de membranas basales capilares y la presencia de IgG, IgM, C3 e IgA de forma irregular y grumosa a nivel del mesangio y capilar con intensa reactividad del mesangio y el subendotelio (membrana basal glomerular), determinaron la presencia de un patrón membranoproliferativo.

Sinembargo, losestudios deinmunofluorescencia revelaron la presencia exclusiva de cadenas ligeras kappa de forma lineal en la membrana basal capilar, la membrana basal tubular y la pared vascular $(+++)$, con depósitos electróndensos dispuestos masivamente en banda a lo largo de las membranas basales capilares, lo cual confirma su carácter monoclonal.

Estos hallazgos, asociados a los cambios descritos en la microscopía electrónica, son característicos de la enfermedad por depósito de cadenas ligeras. En la mayoría de las series, el patrón histológico más frecuente corresponde a una glomerulopatía de tipo nodular, que semeja la de una nefropatía diabética; sin embargo, también es posible encontrar patrones exclusivamente de compromiso túbulointersticial, mesangiocapilar (nuestro caso) o mesangial $(2,3,7,8,12)$. 
En nuestro caso, la cadena depositada en el riñón fue tipo kappa (la presencia en los depósitos de un solo tipo de cadena ligera se consideró indicativa de carácter monoclonal) que, según todas las series, se deposita predominantemente en la enfermedad por depósito de cadenas ligeras, en oposición a lo que ocurre en la amiloidosis, en la que la cadena depositada es lambda (7).

El otro diagnóstico diferencial se establece con la nefropatía por amiloide mediante la tinción rojo Congo, la cual es negativa en los depósitos de cadenas ligeras, y el aspecto diferente en la microscopía electrónica, caracterizado en esta última por depósitos fibrilares (12).

En nuestro paciente, no se detectó un pico monoclonal en sangre ni orina, lo que ocurre en $10 \%$ a $20 \%$ de los casos. Por esta razón, la biopsia renal juega un papel esencial en el diagnóstico de la enfermedad por depósito de cadenas ligeras y en el de la disproteinemia asociada, como queda reflejado en nuestro caso.

Debido a la presencia de enfermedad renal paraproteinémica, se hizo la evaluación hematológica para descartar una neoplasia de células plasmáticas focal, sistémica o generalizada, con depósitos de amiloide, fuera primaria o estuviera asociada a mieloma múltiple o a otras discrasias de las células plasmáticas. Por lo menos, hasta ese momento, se descartó alguna forma de neoplasia sistémica de células plasmáticas (mieloma múltiple) por: falta de incremento en el número de células plasmáticas en médula ósea $(>5 \%)$; ausencia de pico monoclonal en sangre u orina; y falta de otros datos indicativos de una discrasia monoclonal, como serían la presencia de lesiones osteolíticas y la ausencia de depósitos de material amiloide en riñón, recto y grasa abdominal subcutánea mediante la tinción de rojo Congo (13).

El paciente evolucionó hacia una insuficiencia renal rápida y progresiva, con necesidad de terapia renal sustitutiva (diálisis); el tiempo transcurrido desde su primera admisión hasta el inicio de la diálisis fue de 60 días. El tratamiento con quimioterapia, que es indiscutible en pacientes con mieloma, es controvertido cuando no hay una enfermedad maligna (7).
Aunque algunos autores han intentado establecer protocolos para la enfermedad por depósito de cadenas ligeras idiopática, como monoterapia con prednisona, prednisona más melfalan o prednisona más melfalan más plasmaferesis, infortunadamente no pueden sacarse conclusiones sobre la eficacia de estos esquemas de tratamiento, debido al pequeño número de pacientes, a la falta de estandarización de los tratamientos y al limitado tiempo de seguimiento $(7,8)$.

\section{Agradecimientos}

A Jaime Muñoz Carreño por su contribución en la edición final de este trabajo.

\section{Conflicto de intereses}

No existe conflicto de intereses entre los autores, las instituciones y el contenido del presente trabajo.

\section{Financiación}

Declaramos que para la elaboración de este manuscrito no se ha contado con ninguna fuente de financiación.

\section{Referencias}

1. Glenner GG, Terry W, Hurada M, Isersky C, Page D. Amyloid fibril proteins: Proof of homology with immunoglobulin light chains by sequence analyses. Science. 1971;172:1150-1.

2. Ronco P, Plaisier E, Mougenot B, Aucouturier P. Immunoglobulin light (Heavy)-chain deposition disease: from molecular medicine to pathophysiology-driven therapy. Clin J Am Soc Nephrol. 2006;1:1342-50.

3. Ronco PM, Alyanakian MA, Mougenot B, Aucouturier P. Light chain deposition disease: A model of glomerulosclerosis defined at the molecular level. J Am Soc Nephrol. 2001;12:1558-65.

4. Antonovych TT, Lin RC, Parrish E. Light chain deposits in multiple myeloma. Lab Invest. 1974;30:370A.

5. Randall RE, Williamson WC Jr, Mullinax F, Tung MY, Still WJ. Manifestations of systemic light chain deposition. Am J Med. 1976;60:293-9.

6. Ronco P, Aucouturier P, Mougenot B. Kidney involvement in plasma cell dyscrasias. In: Davinson AM, editor. Oxford textbook of clinical nephrology. Third edition. New York, NY: Oxford University Press; 2005.

7. Martín C, Suñer M, Cabrera R, Díaz M, Fernández J. Enfermedad por depósito de cadenas ligeras. Experiencia en nuestro medio. Nefrología. 2008;5:539-42. 
8. Ramos R, Poveda R, Bernís C, Ara J, Sunyer $\mathbf{M}$, Arrizabalaga $\mathbf{P}$, et al. Afectación renal en las gammapatías monoclonales benignas: ¿una entidad infradiagnosticada? Nefrología. 2008;5:525-30.

9. Santostefano M, Zanchelli F, Zaccaria A, Poletti G, Fusaroli M. The ultrastructural basis of renal pathology in monoclonal gammopathies. J Nephrol. 2005;18:65975.

10. Ramos R, Poveda R, Sarrá J, Domingo A, Carreras L, Grinyó JM. Renal involvement in non-malignant IgM gammopathy. Nephrol Dial Transplant. 2007;22;627-30.
11. Sanders PW. Management of paraproteinemic renal disease. Curr Opin Nephrol Hypertens. 2005;14:97-103.

12. García A, Aneiros J, Ramos AM, Petkov V, López JL, Albalate M, et al. Insuficiencia renal por enfermedad de depósito de cadenas ligeras. Nefrología. 2008;2:212-5.

13. Campistol JM. Amiloidosis renal y glomerulonefritis fibrilares. En: Avendaño LH, Aljama $M$, Arias $M$, Caramelo C, Egido JE, Lamas S, editores. Nefrología clínica. Madrid: Editorial Médica Panamericana S.A.; 2003. p. 387-93. 